\title{
Sprachwandel anhand von Produktivitätsverschiebungen in der schweizerdeutschen Derivationsmorphologie
}

\author{
Karina Schneider-Wiejowski (Bielefeld)
}

\begin{abstract}
Claims about productivity of a given affix are often made categorically without any empirical evidence. This paper deals with the question of derivational productivity in a German variety spoken in Switzerland. Although there are a few studies regarding German suffixes and their productivity, it does not exist any study for this special variation of German.

Four German suffixes (-nis, -heit/-keit/-igkeit, -ung and -sal) should be explored in a diachronic way. The question explored is whether there are changes in productivity of these suffixes during time.

There are several possibilities of measuring morphological productivity, by counting frequencies (token and types) and to use probabilistic models. Both methods should be applied in this study. By using the Schweizer Textkorpus it is possible to examine a period of hundred years (1900-2000). It can be shown that there is a morphological change during the 20th century in this German variety. Some of these suffixes become more productive whereas other suffixes lose their efficiency of building new words.
\end{abstract}

\section{$1 \quad$ Einleitung}

Sprachwandel wird insbesondere in der Historischen Linguistik erforscht und bezeichnet den Vorgang der Veränderung und der Entwicklung von Sprache. Dabei ist der Sprachwandelprozess ein sehr langsamer Prozess, der von Sprechern einer Sprache nicht bewusst wahrgenommen wird.

Sprachwandel kann unterschiedliche linguistische Ebenen betreffen; ändern können sich Strukturen auf Wort-, Satz- und Textebene, Bedeutungen und Sprachgebrauchskonventionen auf verschiedenen Ebenen (semantischer Wandel, Bedeutungswandel), Aussprachen auf verschiedenen Ebenen (Lautwandel) und auch orthografische Gegebenheiten. In diesem Aufsatz soll es speziell um den Wortbildungswandel gehen, vielmehr noch um den derivationellen Wandel, der als struktureller Wandel auf Wortebene anzusiedeln ist. Dazu ist es notwendig, kurz auf den Bereich der Morphologie, insbesondere den Bereich der Wortbildung, im Deutschen einzugehen.

Die Morphologie wird in die beiden großen Teildisziplinen Flexion und Wortbildung ${ }^{1}$ eingeteilt. Durch die Flexionsmorphologie werden grammatische Beziehungen zwischen Wortfor-

\footnotetext{
1 Vgl. dazu auch Fleischer/Barz (1995), die darauf aufmerksam machen, dass die Wortbildung deutlich von der Wortschöpfung abzugrenzen ist. Den Unterschied erklären sie dadurch, dass bei der Wortbildung schon vorhandenes Material verwendet wird, was bei der Wortschöpfung nicht der Fall ist. Bei letzterer werden Lautkomplexe geschaffen, die noch nicht als bedeutungstragende Elemente in der jeweiligen Sprache gegeben sind, sodass man von einer Schaffung neuer Wortwurzeln sprechen kann.
} 
men ausgedrückt. Flexionsmorpheme verändern nicht grundsätzlich die lexikalische Semantik der Basis, sondern fügen Informationen hinzu, die die Basis satzfähig machen.

Die Wortbildung lässt sich in die beiden Bereiche Komposition und Derivation aufteilen. ${ }^{2}$ Für diesen Aufsatz ist nur der Bereich der Derivation von Bedeutung. Die Derivation ist dafür verantwortlich, dass neue Lexeme geschaffen werden und das Lexikon erweitert wird. Die meisten Derivate werden mit Affixen gebildet. Dabei ist gerade der Prozess der Präfigierung wie z.B. in an-kaufen oder ver-kaufen als Anhängen eines Affixes vorne an den Wortstamm und der Prozess der Suffigierung wie z.B. wunder-bar oder Bezahl-ung als Anhängen eines Affixes hinter den Wortstamm besonders produktiv. ${ }^{3}$

Im Bereich der Derivation kann es im Laufe der Zeit zu Wandelerscheinungen kommen. ${ }^{4}$ Der Bestand an Wortbildungsmodellen kann sich im Laufe der Zeit verändern. Affixe wie -nis und -sal leben in Bildungen wie Finsternis und Schicksal weiter, untergegangen ist allerdings deren Produktivität, da keine neuen Wörter mit diesen Suffixen gebildet werden. Andere Wortbildungsmodelle, mit denen sie konkurrieren, sind ihnen irgendwann überlegen. Die schon gebildeten Wortbildungsprodukte bleiben dem Sprachbestand allerdings lexikalisiert erhalten.

Wortbildungswandel zeigt sich demnach darin, dass es zu Verschiebungen in der Produktivität kommt. Dieser Zusammenhang soll im 2. Kapitel verdeutlicht werden. Außerdem werden quantitative Methoden zur Messung von Produktivität vorgestellt. Das darauf folgende Kapitel beschreibt die vorliegende Studie, ihren Untersuchungsgegenstand, den Untersuchungszeitraum und ihre Methode. Im vierten Kapitel werden dann die gewonnenen Daten vorgestellt und interpretiert. Das letzte Kapitel, die Diskussion, fasst noch einmal zusammen, was mit dieser Untersuchung gezeigt werden konnte, und zeigt auf, welche Forschungsmöglichkeiten sich in diesem Bereich noch bieten.

\section{Wortbildungswandel und Produktivität}

Der Wortbildungswandel wurde bisher wenig erforscht. Vielmehr fokussierte man in der Vergangenheit auf den Lautwandel, der durch Regularitäten erklärt werden kann.5

Der Wortbildungswandel ist ein Phänomen des Sprachwandels, das sich anhand von Produktivitätsverschiebungen verdeutlichen lässt. Wird ein Affix produktiver, so lassen sich viele Neubildungen mit diesem Affix konstruieren, die Produktivität steigt. Das Absinken der Produktivität führt dazu, dass ein Wortbildungsmodell nicht mehr angewendet wird, um Neubildungen zu schaffen. Obwohl das Phänomen der Produktivität in der Derivationsmorphologie erst insbesondere in Zeiten der Korpuslinguistik populär geworden ist, spricht schon Wilmanns (1899) im Bezug auf Ableitungstypen von "Lebenskraft und Dauer" und beleuchtet wenn auch nur ansatzweise - den Zusammenhang von Wortbildungsprozessen und ihrer Veränderung. Diese diachrone Veränderung wird in vielen neueren Arbeiten ausgiebig behandelt (z. B. in Bauer 2001, Demske 2000, Kiesewetter 1987 oder Cowie 1999, Scherer 2005).

Das Derivationssystem der schweizerdeutschen Varietät hingegen ist bislang nicht in den Fokus der wissenschaftlichen Forschung geraten. Diese empirische Lücke soll mit diesem Aufsatz geschlossen werden. Dabei wird das Schweizer Textkorpus verwendet, das die Möglichkeit bietet, die schweizerdeutsche Sprache von 1900-2000 zu untersuchen. Dabei ist das Schweizer Textkorpus bisher noch im Aufbau. Es soll nach der Fertigstellung 20 Millionen

\footnotetext{
2 Zu den Grundlagen der Wortbildung siehe auch Eisenberg (2006), Erben (2000) und Lohde (2006).

${ }^{3}$ Siehe dazu auch Bauer (2007), der darauf aufmerksam macht, dass die Suffigierung in den meisten Sprachen weitaus häufiger verwendet wird als die Präfigierung.

${ }^{4}$ Siehe dazu Habermann et al. (2002).

5 Siehe dazu beispielsweise Andersen (1980).
} 
Textwörter beinhalten. 6 Zum Zeitpunkt der Untersuchung (März 2009) waren etwa drei Viertel der zu digitalisierenden Texte online verfügbar. Die Nutzung dieses Korpus ist komfortabel, da es unter anderem eine Filter- und Exportfunktion besitzt.

Das Korpus ist ein Teil eines internationalen Forschungsvorhabens, das die Erfassung der deutschen Standardsprache des 20. Jahrhunderts zum Ziel hat. Es vereint deutschsprachige Texte von Schweizer Autoren aus dem 20. Jahrhundert. Die digitale Sammlung ist analog zu den anderen Teilkorpora in Deutschland, Italien und Österreich anhand formaler, inhaltlicher und zeitlicher Kriterien aufgebaut. Die Besonderheit ist die ausgewogene Repräsentation des Deutschschweizer Wortschatzes. ${ }^{7}$

Anhand dieses Korpus sollen unterschiedliche Derivationssuffixe hinsichtlich ihrer Produktivität miteinander verglichen werden. Wie diese Vergleichbarkeit durch Anwendung eines quantitativen Maßes hergestellt wird, soll im Kap. 2.1 erläutert werden.

Neben der Vergleichbarkeit der unterschiedlichen Suffixe untereinander wird es in diesem Aufsatz auch um die diachrone Veränderung der einzelnen Suffixe im Laufe des untersuchten Zeitraums gehen. Dabei steht die Frage im Zentrum, wie es sich mit der Produktivität der Suffixe verhält; ob es zu einem Ansteigen, einem Gleichbleiben oder einem Abfall in der Produktivität kommt.

\subsection{Methoden zur Messung von Produktivität}

Das Phänomen der Produktivität in der Morphologie ist erst in den letzten 30 Jahren intensiv beschrieben und untersucht worden. So sagt z.B. Mayerthaler (1981: 124), der den Begriff der Produktivität beschreibt, dass er "zu den unklarsten Begriffen der Linguistik" gehöre. Und gerade für den Bereich der Derivation schien es da - vor nicht allzu langer Zeit - noch einige Unklarheiten zu geben. ${ }^{8}$

Für die Messung von Produktivität gibt es unterschiedliche methodische Ansätze; bewährt haben sich vor allem bestimmte Frequenz- und Wahrscheinlichkeitsmodelle. Einige dieser sollen in den beiden folgenden Kapiteln vorgestellt werden.

\subsubsection{Frequenzmodelle}

Will man Häufigkeiten von Suffixen untersuchen, so bietet es sich an, Tokens oder Types unterschiedlicher Affixe miteinander zu vergleichen.

Beispielsweise kann das englische Suffix -ric nur mit einem einzigen Morphem verbunden werden, sodass das Wort bishopric entsteht. Die Typehäufigkeit ist dementsprechend genau 1. Anders sieht es beispielsweise bei dem Suffix -bar aus, für das die Recherche in einem digitalen Lexikon ${ }^{9} 1015$ Treffer anzeigt.

Auch die Tokenhäufigkeit kann Aufschluss darüber geben, ob ein Suffix produktiv ist, indem es viele Wortbildungen hervorbringt. ${ }^{10}$

\footnotetext{
${ }^{6}$ Zur genauen Beschreibung des Korpus und seiner Nutzungsfunktion siehe Bickel et al. (in diesem Band, Heft 2).

${ }^{7}$ Siehe dazu http://www.dwds.ch.

${ }^{8}$ Siehe dazu Aronoff (1976: 36): "Productivity is one of the central mysteries of derivational morphology".

9 http://www .owid.de/ (25.01.2009).

10 Token- und Typehäufigkeit können auch interagieren, sodass es auch geläufig ist, diese beiden Werte in Relation zueinander zu stellen. Dieses soll aber in diesem Aufsatz unberücksichtigt bleiben.
} 


\subsubsection{Wahrscheinlichkeitsmodelle}

Die Anwendung von Wahrscheinlichkeitsmodellen zur Ermittlung von Produktivität soll es erlauben, Aussagen über die Entwicklung von Produktivität zu machen, indem man statistische Werte interpretiert. Dabei geht es um eine Kombination verschiedener Häufigkeiten.

Probabilistic models rely largely on Baayens research (1992, 1993, Baayen and Lieber 1991), which develops from Aronoffs principle that token frequency is related to semantic complexity. Thus, token frequency is central to Baayens models. Specifically, his computations are based on the notion of hapax legomena. (Fernández-Domínguez et al. 2007: 38).

Es gibt, so Baayen (1992), zwei Maße, die angewendet werden können, um Produktivität zu errechnen: $P$ und $P^{*}$. Beide von ihm vorgeschlagenen Maße sollen nun vorgestellt werden.

Baayens Produktivitätsindex P basiert auf der Anzahl der sogenannten Hapax Legomena und der Anzahl der Tokens. Dabei repräsentieren die Hapax Legomena jene Formen mit dem zu untersuchenden Affix, die nur ein einziges Mal vorkommen, da es sich mit großer Wahrscheinlichkeit um neugebildete Formen handelt. Der im Nenner stehende Wert, der sich aus der Anzahl der Tokens ergibt, zeigt dabei die komplette Anzahl der gebildeten Formen, die das Affix beinhalten:

$$
P=\frac{V(N)}{N}
$$

Zusammenfassend zu dieser Methode lässt sich demnach sagen, dass die Wahrscheinlichkeit zur Neubildung von Wörtern mit einem bestimmten Affix im Vordergrund steht. Ein Affix, das produktiv ist, wird folglich viele Neubildungen hervorbringen, sodass ein hoher Wert P entsteht.

Dieses Maß lässt sich dabei sowohl dazu verwenden, unterschiedliche Suffixe hinsichtlich ihrer Produktivität untereinander zu untersuchen, als auch, um den Wandel eines einzigen Suffixes zu betrachten. Unter diachroner Perspektive könnte man einzelne Zeitpunkte untersuchen, die dann im Zusammenhang interpretiert werden können.

\section{$3 \quad$ Korpuslinguistische Untersuchung}

In diesem Kapitel sollen die Rahmenbedingungen der Untersuchung vorgestellt werden. Dabei geht es in Kap. 3.1 um die Beschreibung der zu untersuchenden Derivationssuffixe, in Kap. 3.2 um die Definition des Untersuchungszeitraums und in 3.3 wird die Methode der Auswertung erläutert.

\subsection{Untersuchungsgegenstand}

Untersucht werden vier unterschiedliche Derivationssuffixe: -nis, -heit (-keit/-igkeit), -sal und -ung, die im Folgenden kurz beschrieben werden sollen.

Das Suffix -nis bildet feminine oder neutrale Abstrakta. Heutzutage bezeichnet man es nicht mehr als produktiv. ${ }^{11}$ Am häufigsten dient eine (präfigierte) verbale Basis zur Suffigierung, sodass es zur Bildung von Wörtern wie z.B. Befugnis, Erlaubnis, Ereignis oder Bedürfnis gekommen ist. Semantisch gesehen kam es durch eine Derivation mit -nis zu Vorgangsbeschreibungen oder Beschreibungen von Zuständen. Wichtig ist an dieser Stelle zu erwähnen, dass es oftmals daneben ein -ung-Derivat gibt, das den Prozesscharakter als Abstraktum stärker zum Ausdruck bringt.

Als Erklärung für diese Art von parallel existierenden Wörtern (Erzeugung - Erzeugnis, Hemmung - Hemmnis ...) nennt Shippan (1967: 80) die Unfähigkeit seitens der -nis-Derivate,

\footnotetext{
${ }^{11}$ Vgl. dazu Fleischer/Barz (1995).
} 
objektive Genitive an sich zu binden. In der deutschen Sprache gibt es sowohl Paare dieser Art, die sich semantisch stärker voneinander unterscheiden, als auch Wörter, die synonym zueinander sind, wie es beispielsweise bei Verlobung und Verlöbnis der Fall ist. Auch bei den -nis-Derivaten kann eine substantivische Basis Ausgangspunkt gewesen sein. Dieses ist allerdings eher selten und schon gar nicht produktiv.

Ebenso verhält es sich mit der adjektivischen Basis. Auch hier kann angemerkt werden, dass es Parallelbildungen auf -nis gibt, z. B. in Bitternis - Bitterkeit.

Der Ursprung für das deutsche Suffix -heit geht auf ein mittelhochdeutsches Substantiv heit zurück, das vor seiner Grammatikalisierung 'Art und Weise, Beschaffenheit, Eigenschaft, Person, Stand' beschrieben hat. Das Suffix kann zum einen eine adjektivische Basis besitzen und dann in drei Varianten (-heit, -keit, -igkeit), Allomorphen, erscheinen. Die drei Varianten sind distributiv zueinander, und auch wenn es sicherlich Überlappungen gibt, sind die Vorkommensverhältnisse recht klar, da es Unterschiede im Akzentmuster gibt. Schon Wilmanns (1899: 386) stellt fest, dass die Variante -keit hinter einer unbetonten Silbe stehen muss. Aus diesem Grund bietet es sich an, suffigierte Basen auf -bar, -ig, -lich, -isch oder -sam zu verwenden. Aber es verbindet sich auch mit simplizischen Basen, wie es z.B. bei Heiser- der Fall ist. Die Variante -igkeit findet sich nach Basen auf -ig und auch nach Basen auf -haft und -los. Außerdem lassen sich im Deutschen auf - $e$ auslautende Adjektive mit -igkeit suffigieren. Bei Suffigierungen mit -heit gibt es, so Fleischer/Barz (1995: 160), sehr unterschiedliche Akzentmuster. Zwischen den drei Variationen gibt es aus semantischer Sicht keine Unterschiede. Durch sie werden "substantivische Eigenschaftsbezeichnungen" und Verhaltensweisen und Handlungen geschaffen.

Das Suffix -sal ist, so Fleischer/Barz (1995: 168), heute unproduktiv. Es erscheint nur noch in einigen Substantiven, vor allem mit verbaler und simplizischer Basis wie z. B. in Labsal oder Rinnsal.

Das Suffix -ung ist das produktivste substantivbildende Suffix im Deutschen. 1970 existieren, so Mater (1970), etwa 9575 Derivate auf -ung. Fleischer/Barz (1995: 172) sprechen von bei den -ung-Derivaten von einer semantisch offenen und wenig festgelegten Bildungsweise. Die Bildung einer Substantivierung hat oftmals eine verbale Basis. Dieses können Simplizia sein, wie es z.B. in Glätt- oder Spaltung der Fall ist. Auch Präfixverben wie in Einbild- oder in Verbindung bieten eine optimale Basis. Diese Verben können auch doppelt präfigiert sein. So ist es im Deutschen möglich, anerkenn- oder verabred- zu suffigieren. Eine genaue Auflistung aller Möglichkeiten, zu denen beispielsweise auch noch kombinatorische Derivate, verbale Komposita und verbale Wortgruppen gehören, findet sich in Fleischer/Barz (1995: 172)..$^{12}$

So sehr man bei diesem Suffix auch von Produktivität sprechen kann, so gibt es dennoch auch Vorzugsbildungen und Einschränkungen. Das Derivat von einem verbalen Simplex ist im Deutschen manchmal nicht geläufig: Anhörung - *Hörung. Auch gibt es Einschränkungen hinsichtlich der präfigierten Verben; an der Stelle, an der es geläufige Simplizia gibt, wie es beispielsweise bei Abgabe der Fall ist, wird man abgeb* nicht $\mathrm{zu} *$ Abgebung umwandeln. Neben der verbalen Basis kann auch eine substantivische Basis als Ausgangspunkt dienen. Dieses Modell ist aber, so Fleischer/Barz (1995: 176), nur noch schwach produktiv. Dennoch findet man in der deutschen Gegenwartssprache Wörter, die nach diesem Muster gebildet worden sind. Das Wortbildungsmuster mit einer adjektivischen Basis ist unproduktiv. ${ }^{13}$

\footnotetext{
12 Zur Produktivität der beschriebenen Suffixe siehe auch Motsch (2004:19), der von "aktiven" und "inaktiven" Wortbildungsmustern spricht. Dabei hält er -nis für inaktiv und -heit/-keit/-igkeit und -ung für stark aktiv. $\mathrm{Zu}-$ sal wird keine Aussage gemacht.

${ }^{13}$ Dennoch belegen Fleischer/Barz (1995: 176) das Vorkommen dieses Musters anhand der Wörter Dickung, Festung oder auch Niederung.
} 
Als vierte Basis von -ung-Derivaten listen Fleischer/Barz (1995) die Gruppe der isolierten Bildungen auf. Motivationsbeziehungen zum Grundmorphem lassen sich nur schwer herstellen, da die Basis oftmals nicht mehr im Gebrauch ist oder es sich um eine noch nicht alte Entlehnung handelt. Innung z. B. lässt sich auf das mittelhochdeutsche innen zurückführen, was so viel heißt wie 'in einen Verband aufnehmen'.

\subsection{Untersuchungszeitraum}

Das Schweizer Textkorpus bietet die Möglichkeit, die schweizerdeutsche Varietät über einen Zeitraum von 1900 bis 2000 zu untersuchen. Denkbar wäre demnach ein Produktivitätsvergleich der Suffixe in diesem Zeitraum.

Interessant ist dabei aber nicht nur die Frage nach den einzelnen Produktivitätsgraden der Suffixe untereinander, sondern auch die Frage nach Veränderungen der einzelnen Derivationsmodelle innerhalb der 100 Jahre. Um eine diachrone Studie dieser Art durchzuführen, ist es notwendig, mehrere Messzeitpunkte zu wählen. In dieser Studie wurden die fünf Zeitpunkte $1900,1925,1950,1975$ und 1999 gewählt.

\subsection{Methode der Auswertung}

Das Schweizer Textkorpus beinhaltet eine Filterfunktion, die es ermöglicht, mit regulären Ausdrücken (z. B. *nis) nach einzelnen Wortbildungen innerhalb eines festgelegten Zeitraums zu suchen. Man erhält eine Konkordanzliste, die dann (teil)automatisch weiter verarbeitet werden kann.

In dieser Studie sollen drei unterschiedliche Maße zur Beschreibung von Produktivität betrachtet werden:

- Tokenhäufigkeit

- $\quad$ Typehäufigkeit

- P. 


\section{$4 \quad$ Ergebnisse}

$\mathrm{Zu}$ allererst soll die Token- und Typehäufigkeit und die Anzahl der Hapax legomena betrachtet werden. Die erhobenen Daten ergeben dabei die folgenden Frequenzen:

\begin{tabular}{|l|c|c|c|c|c|c|}
\hline & \multicolumn{3}{|c|}{-heit (-keit/-igkeit) } & \multicolumn{3}{c|}{-ung } \\
\hline & Tokens & Types & Hapax l. & Tokens & Types & Hapax l. \\
\hline $\mathbf{1 9 0 0}$ & 530 & 210 & 110 & 1477 & 381 & 96 \\
\hline $\mathbf{1 9 2 5}$ & 645 & 207 & 132 & 2654 & 446 & 148 \\
\hline $\mathbf{1 9 5 0}$ & 1047 & 329 & 214 & 2645 & 453 & 141 \\
\hline $\mathbf{1 9 7 5}$ & 1290 & 257 & 264 & 2207 & 391 & 172 \\
\hline $\mathbf{1 9 9 9}$ & 739 & 183 & 153 & 3017 & 521 & 365 \\
\hline & & - -nis & & & -sal & \\
\hline & Tokens & Types & Hapax l. & Tokens & Types & Hapax l. \\
\hline $\mathbf{1 9 0 0}$ & 151 & 32 & 11 & 7 & 2 & 1 \\
\hline $\mathbf{1 9 2 5}$ & 270 & 30 & 12 & 16 & 3 & 1 \\
\hline $\mathbf{1 9 5 0}$ & 321 & 35 & 10 & 24 & 3 & 1 \\
\hline $\mathbf{1 9 7 5}$ & 434 & 30 & 9 & 10 & 1 & 0 \\
\hline $\mathbf{1 9 9 9}$ & 263 & 20 & 4 & 7 & 1 & 0 \\
\hline
\end{tabular}

Abb. 1: Token- und Typehäufigkeit und Anzahl der hapax legomena bei den Suffixen -heit (-keit/-igkeit), -ung, -nis und -sal

Anhand der Tabelle wird deutlich, dass sich die einzelnen Suffixe im Hinblick auf ihre Produktivität unterschiedlich verhalten, was sich in den Frequenzen niederschlägt.

Unter Betrachtung von Tokens und Types erscheint -ung zu allen fünf Messzeitpunkten am produktivsten, indem es die meisten Wortformen $(1477,2654,2645,2207,3017)$, und auch Types $(210,207,329,257,183)$ hervorbringt. Demzufolge ist auch die zu den anderen Suffixen vergleichbar hohe Anzahl an hapax legomena $(96,148,141,172,365)$ nicht verwunderlich.

An zweiter Stelle folgen die Allomorphe -heit, -keit und -igkeit, die zwar verglichen mit -ung viel weniger Bildungen hervorbringen, aber dennoch als produktiv bezeichnet werden können, da Token- und Typeanzahl noch relativ hoch sind.

Die Token- und Typehäufigkeit von -nis und -sal zeigt, dass beide Suffixe als unproduktiv angesehen werden können, da nur wenige Wörter mit diesen Suffixen gebildet werden. Dennoch unterscheiden sich auch diese beiden Suffixe enorm; während es mit -nis immer noch zwischen 20 und 35 Bildungen gibt, findet man kaum noch Bildungen mit - sal. ${ }^{14}$

Im Bezug auf die Struktur des Ausschnitts des schweizerdeutschen Derivationssystem lässt sich sagen, dass diese Struktur während der gesamten 100 Jahre erhalten bleibt; schaut man sich die Frequenzen an, so wird man für alle Zeitpunkte die folgende Rangfolge aufstellen können: 1. -ung, 2. -heit (-keit/-igkeit), 3. -nis und 4. -sal. Als Veranschaulichung kann die folgende Abbildung hinzugezogen werden, die die Frequenzen der einzelnen Suffixe von 1900 aufzeigt und dabei auch als repräsentative Struktur für alle untersuchten Zeitpunkte gelten kann:

\footnotetext{
14 Bei den Belegen handelt es sich um die Bildungen Mühsal, Schicksal und Rinnsal.
} 


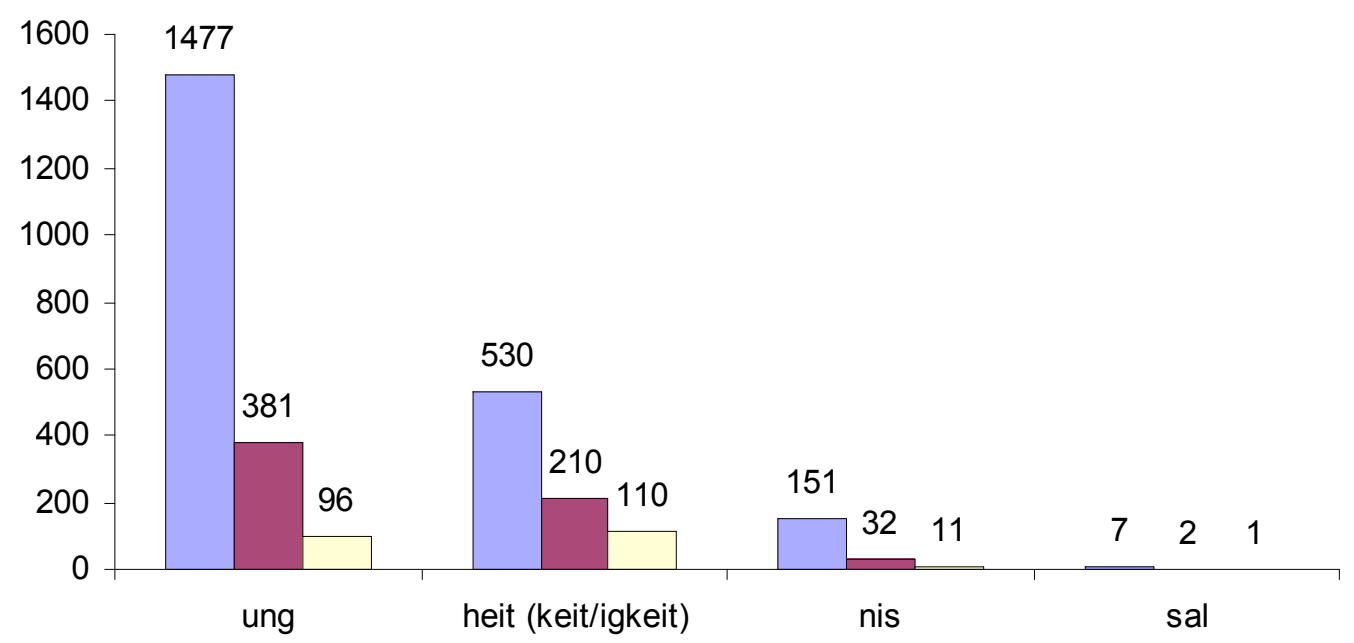

Abb. 2: Frequenzen von Token, Types und Hapax Legomena der Suffixe -ung, -heit (-keit/-igkeit), -nis und -sal 1900

Doch wie schon in Kapitel 2 erwähnt, sind Token- und Typehäufigkeit nicht die einzigen MaBe, die zur Betrachtung der Produktivität dienen. Vielmehr ist es interessant, die einmaligen Bildungen mit den gesamten Bildungen mit einem Suffix in Relation zueinander zu setzen, indem man Baayens Formel anwendet und $\mathrm{P}$ berechnet:

\begin{tabular}{|c|c|c|c|c|}
\hline & -heit (-keit/-igkeit) & -ung & $-n i s$ & $-s a l$ \\
\hline & \multicolumn{4}{|l|}{$\mathbf{P}$} \\
\hline 1900 & 0.21 & 0.065 & 0.072 & 0.14 \\
\hline 1925 & 0.21 & 0.056 & 0.044 & 0.063 \\
\hline 1950 & 0.2 & 0.053 & 0.031 & 0.042 \\
\hline 1975 & 0.2 & 0.078 & 0.02 & 0 \\
\hline 1999 & 0.21 & 0.12 & 0.015 & 0 \\
\hline
\end{tabular}

Nun stellt sich auch die Frage nach den Veränderungen bei den einzelnen Suffixen. 
Um den Produktivitätsverlauf zu veranschaulichen, soll die folgende Grafik betrachtet werden:

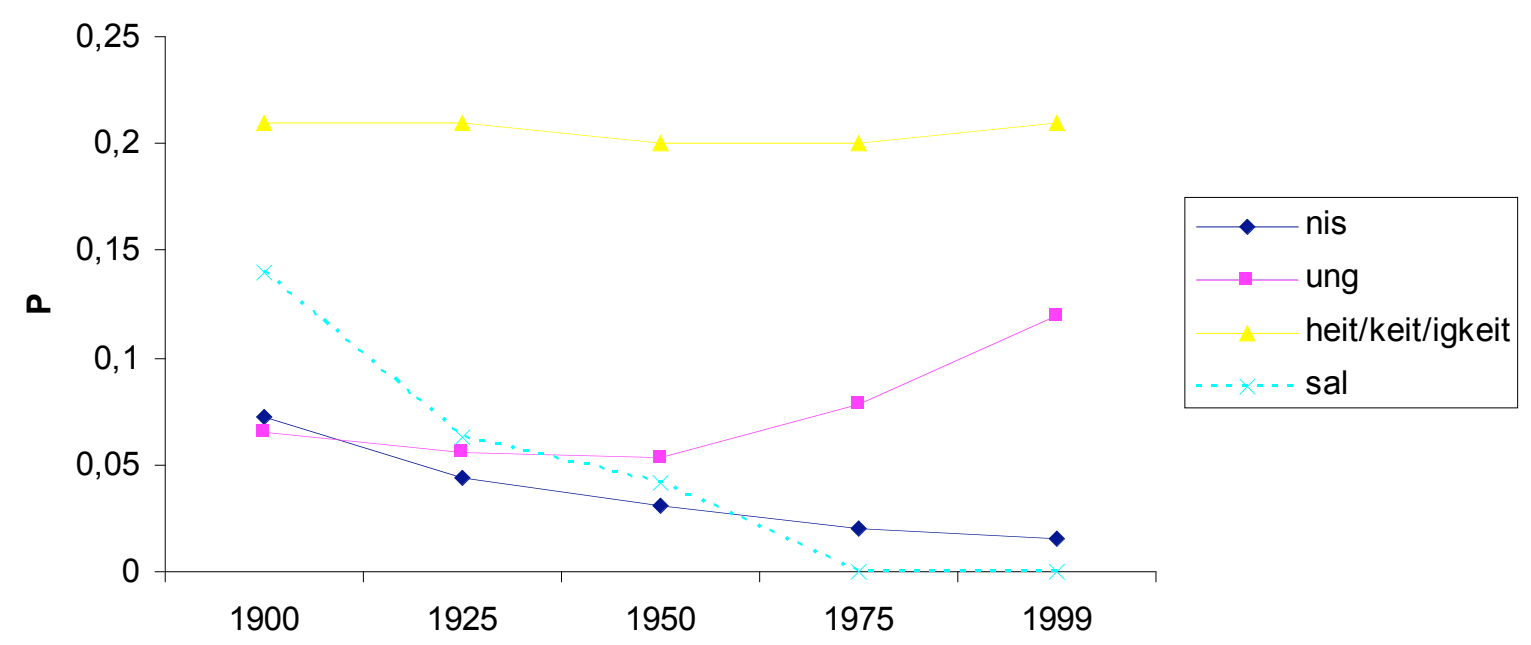

Abb. 3: Produktivitätsverläufe von -heit (-keit/-igkeit), -ung, -nis und -sal im 20. Jahrhundert

Anhand der Grafik (und der Tabelle) wird ersichtlich, dass das Suffix -heit mit seinen Allomorphen zu allen Zeitpunkten die größte Produktivität nach P besitzt. Das Suffix -ung ist trotzt hoher Frequenzen der Tokens und Types unproduktiver als die Allomorphe von -heit. Dennoch macht dieses Suffix auf der anderen Seite die stärkste Entwicklung durch. Ab 1950 steigt die Produktivität von -ung stark an. Das Suffix -heit hingegen bleibt während des gesamten Untersuchungszeitraums sehr konstant.

$\mathrm{Zu}$-nis kann gesagt werden, dass es nur als sehr schwach produktiv bezeichnet werden kann. Außerdem wird anhand der Werte deutlich, dass die Produktivität im Laufe der untersuchten Jahre stetig sinkt.

Schaut man sich die Werte und die Entwicklung von dem als unproduktiv beschriebenen Suffix -sal an, so wird man auf den ersten Blick feststellen, dass es insbesondere um 1900 als produktiv bezeichnet werden müsste. Der Wert von 0.14 ist damit sogar höher als der Wert von -ung oder -nis. Der Verlauf der Kurve würde vermuten lassen, dass -sal innerhalb der nächsten 75 Jahre an Produktivität verliert und ab 1975 nicht mehr zur Wortbildung beiträgt. Doch dieser Verlauf ist als kritisch zu betrachten, da sich die teils hohen Werte von P aus sehr niedrigen Frequenzen ergeben. Ein Suffix, das produktiv ist, wird nicht nur 7 Tokens und 2 Types hervorbringen, wie es um 1900 der Fall ist. Dass es dennoch Wörter gibt, die nur einmal vorkommen, liegt allerdings nicht daran, dass es Neubildungen mit dem Suffix -sal gibt, sondern daran, dass die einmalig vorkommenden Bildungen schon so veraltet sind, dass sie nur noch sehr selten verwendet werden.

\section{$5 \quad$ Diskussion}

In dieser korpuslinguistischen Untersuchung ging es um die Beantwortung der Frage nach Wandelerscheinungen in der Derivationsmorphologie der schweizerdeutschen Varietät, um die Frage nach einem Wortbildungswandel im Zeitraum von 1900 bis 2000.

Das Schweizer Textkorpus diente dabei als Datengrundlage. Extrahiert wurden alle Derivate auf -ung, -heit (-keit/-igkeit), -sal und -nis zu den Zeitpunkten 1900, 1925 1950, 1975 und 2000. Dabei ging es zum einen darum, die deskriptiven und sehr kategorischen Aussagen der linguistischen Literatur statistisch zu belegen, wie produktiv die einzelnen Derivationssuffixe 
zur Wortbildung beitragen, und zum anderen sollten Veränderungen aufgezeigt werden, an denen sich ein Wortbildungswandel manifestiert und die auch statistisch messbar sind.

Zur Beschreibung und Berechnung von Produktivität können zwei unterschiedliche Methoden angewendet werden, Frequenzmodelle und statistische Maße, die auf Baayen (1992) zurückgehen. Beide Methoden wurden in dieser Studie angewandt. Schaut man sich die einzelnen Frequenzen an, so erscheint das Suffix -ung das produktivste der vier untersuchten zu sein. Danach folgen die Allomorphe von -heit. Kaum produktiv erscheint -nis, und -sal ist als unproduktiv zu bezeichnen. Die Struktur der Rangfolge bleibt über den gesamten Untersuchungszeitraum erhalten.

Nach Anwendung des Maßes P, eines Wahrscheinlichkeitsmodells, ergibt sich allerdings eine andere Rangfolge hinsichtlich der Produktivität. Den ersten Platz belegt nicht mehr das Suffix -ung, sondern die Allomorphe von -heit. Das Suffix

-ung weist allerdings auch eine hohe Produktivität auf. Zu -nis und -sal kann gesagt werden, dass die gleiche Interpretation erfolgen kann wie sie auch nach Betrachtung der Frequenzmaße gegeben ist; -nis ist als schwach produktiv zu betrachten und -sal kann als unproduktiv bezeichnet werden. Schaut man sich die einzelnen Suffixe unter diachroner Perspektive an und betrachtet den Verlauf der Produktivität, so wird man feststellen, dass das Suffix -ung dasjenige Suffix ist, das innerhalb der hundert Jahre an Produktivität gewinnt. Der Anstieg erfolgt allerdings erst im Zeitraum 1950 bis 2000. Bei -heit/-keit/-igkeit lässt sich feststellen, dass die Produktivität stets konstant bleibt. Der Wert von etwa 0.2 ändert sich innerhalb des Untersuchungszeitraums nicht. $\mathrm{Zu}$-nis ist zu sagen, dass sich ein Produktivitätsabfall beobachten lässt. Der Wert sinkt innerhalb des Untersuchungszeitraums konstant ab. Es lässt sich demnach vermuten, dass das Wortbildungsmodell mit dem Suffix -nis auch in den nächsten Jahren an Produktivität verlieren wird. Ob die Produktivität des Suffixes -nis so weit sinkt, dass es völlig unproduktiv wird und nicht mehr zur Neubildung von Wörtern beiträgt (wie dies bei -sal geschehen ist), wäre eine interessante Untersuchungsfrage für die künftige linguistische Forschung. Das Affix -sal ist absolut unproduktiv, was sich sowohl in den Frequenzen als auch im Wert $P$ ausdrückt.

Im Bezug auf die Produktivitätsmethoden ist eine weitere Frage zu beantworten, die nach der Reliabilität dieser Methoden. Man muss sich überlegen, welche Maße man anwendet, um Produktivitätsveränderungen zu betrachten. Die älteren der beiden Methoden ist die Betrachtung der Frequenzen (Tokens und Types). Insbesondere die Methode der Typehäufigkeit wurde in Zeiten vor der Computerlinguistik durchgeführt in sogenannten Wörterbuchstudien. Eine hohe Typeanzahl bestätigt, dass sich ein Suffix an viele Basen binden kann, sodass viele Wörter nach diesem Wortbildungsmodell gebildet worden sind.

Doch das Problem, das durch die Typezählung entsteht, ist, dass nicht deutlich werden kann, ob ein Wortbildungsmuster auch zur Neubildung führt, was ein wichtiges Kriterium in der Produktivitätsmessung darstellt.

Um den Aspekt der neugebildeten Wörter mit einzubeziehen, bietet es sich an, Baayens Formel $\mathrm{P}$ anzuwenden und die einmaligen mit einem Suffix gebildeten Formen in Relation zu der Gesamtanzahl aller Bildungen zu setzen. Die Interpretation der Werte erlaubt es, Aussagen darüber zu machen, wie hoch die Wahrscheinlichkeit ist, dass neue Wörter mit einem bestimmten Suffix gebildet worden sind.

Doch beide methodischen Ansätze - die Frequenzbetrachtung und die Wahrscheinlichkeitsberechnung - müssen sich nicht gegenseitig ausschließen. Will man Produktivität untersuchen, so bietet es sich an, beide Methoden anzuwenden. Danach stellt sich allerdings die Frage nach der Gewichtung. Da die Wahrscheinlichkeitsberechnung mit P den Hauptaspekt der Neubildung mit einbezieht, sollte diese Methode die bevorzugte von beiden sein. Dennoch 
darf, und das lässt sich an dem Suffix -sal zeigen, nicht nur die reine Zahl P interpretiert werden, vielmehr müssen auch die Häufigkeiten bei der Überprüfung der jeweiligen Werte in Betracht gezogen werden, denn ein hoher Wert $\mathrm{P}$ kann sich auch aus niedriger Tokenfrequenz und geringer Anzahl der Hapax legomena ergeben. Demnach lässt sich auch daraus folgern, dass die Berechnung des Wertes P nur dann einen Sinn ergibt, wenn eine gewisse Anzahl an Tokens vorhanden ist. Bei einer Tokenanzahl zwischen 7 und 24, wie es für -sal festgestellt wurde, konnten auch Wörter gefunden werden, die einmalig vorkommen. Doch schaut man sich diese einmalig belegbaren Wörter an - es handelt sich dabei einmal um Rinnsal, einmal um Trübsal und in einem anderen Jahr um Mühsal -, so muss gesagt werden, dass es sich in keinem der Fälle um Neubildungen handelt, sondern um sehr veraltete Bildungen, die einfach nicht mehr häufig im Sprachgebrauch zu finden sind. Eine Aussage über Produktivität oder Unproduktivität und auch Tendenzen dieser lässt sich in diesem Fall deshalb nur anhand der Token- und Typefrequenz machen. Diese Studie konnte demnach zeigen, dass es durchaus sinnvoll ist, mehrere unterschiedliche Maße zu verwenden. Außerdem kann gesagt werden, dass es im Bereich der derivationellen Morphologie noch einiges gibt, was zu erforschen ist. Interessant wäre eine Erhebung weiterer Daten im Bezug auf noch andere Derivationssuffixe. Auch das Präfixsystem könnte durch eine derartige Analyse betrachtet werden. Durchaus sinnvoll wäre es auch, andere Korpora ähnlichen Aufbaus zu betrachten, um zu vergleichen, ob die Ergebnisse stabil sind.

Weiterhin könnte das Ergebnis, das für das Schweizerdeutsche festgestellt werden konnte, mit anderen deutschen Varietäten oder anderen germanischen Sprachen verglichen werden. Es darf also festgehalten werden, dass der Forschungsspielraum auf dem Gebiet der derivationellen Morphologie im Hinblick auf ihre Produktivität noch sehr groß ist und dass es noch viele offene Fragen gibt, die zu beantworten sind.

\section{Literaturangaben}

Aronoff, Mark (1976): Word Formation in Generative Grammar. Cambridge, Mass.: MIT Press.

Baayen, Harald (1989): A Corpus-Based Approach to Morphological Productivity: Statistical Analysis and Psycholinguistic Interpretation. Amsterdam, Ph.D. thesis, Free University.

Baayen, Harald (1992): "Quantitative aspects of morphological productivity". Yearbook of Morphology 1992: 109-149.

Baayen, Harald (1993): "On frequency, transparency and productivity". Yearbook of Morphology 1992: 181-208.

Baayen, Harald (1994): "Derivational productivity and text typology". Journal of quantitative linguistics 1: 16-34.

Bauer, Laurie (2001): Morphological Productivity. Cambridge: Cambridge University Press.

Bauer, Laurie (2007): "Derivational morphology". Language and Linguistics Compass 2/1: 196-210.

Cowie, Claire (1999): Diachronic word-formation: a corpus-based study of derived nominalizations in the history of English. Cambridge, Univ., Ph.D. thesis.

Demske, Ulrike (2000): "Zur Geschichte der ung-Nominalisierung im Deutschen. Ein Wandel morphologischer Produktivität". Beiträge zur Geschichte der deutschen Sprache und Literatur 3/122: 365-411.

Eisenberg, Peter (2006): Grundriss der deutschen Grammatik. Band 1: Das Wort. 3., durchgesehene Auflage. Stuttgart/Weimar: Metzler.

Erben, Johannes (2000): Einführung in die deutsche Wortbildungslehre. 4. aktualisierte und ergänzte Auflage. Berlin: Erich Schmidt.

Fleischer, Wolfgang/Barz, Irmhild (1995): Wortbildung der deutschen Gegenwartssprache. 2. Auflage. Tübingen: Niemeyer. 
Fernández-Domínguez, Jesús/Díaz-Negrillo, Ana/Štekauer, Pavol (2007): "How is low morphological productivity measured?" Atlantis, revista de la Asociación Española de Estudios Anglo Norteamericanos 1/29: 29-54.

Habermann, Mechthild/Müller, Peter O./Munske, Horst Haider (eds.) (2002): Historische Wortbildung des Deutschen. Tübingen: Niemeyer. (= Germanistische Linguistik 232).

Kiesewetter, Jutta (1987): Entwicklungsprozesse in der deutschen Wortbildung, in Ausschnitten verfolgt und dargestellt an den verbalen Bildungen mit den Elementen bei-, dar-, und -ob. Leipzig, Univ., Diss.

Mayerthaler, Willi (1981): Morphologische Natürlichkeit. Wiesbaden. Aula.

Motsch, Werner (2004). Deutsche Wortbildung in Grundzügen. 2. überarbeitete Auflage. Berlin: de Gruyter.

Scherer, Carmen (2005): Wortbildungswandel und Produktivität. Eine empirische Studie zur nominalen -er-Derivation im Deutschen. Tübingen: Niemeyer.

Shippan, Thea (1967): Die Verbalsubstantive der deutschen Sprache der Gegenwart. Habilitationsschrift, Karl-Marx-Universität Leipzig.

Wilmanns, Wilhelm (1899): Deutsche Grammatik. 2. Abteilung: Wortbildung. Straßburg: Trübner. 